\title{
Sorting, identification and enrichment of side population cells in THP-1 acute monocytic leukemia cells
}

\author{
YINGCHAO WANG, CHUYUN YIN, LEI FENG, LINA MA, YONGWEI WEI and GUANGYAO SHENG \\ Department of Pediatrics, The First Affiliated Hospital of Zhengzhou University, Zhengzhou, Henan 450052, P.R. China
}

Received November 12, 2012; Accepted January 30, 2013

DOI: $10.3892 /$ or.2013.2316

\begin{abstract}
The objective of the present study was to examine and determine whether the human acute monocytic leukemia cell line THP-1 contains side population (SP) cells, and, if so, to increase the proportion of SP cells using arabinosylcytosine (Ara-C). Fluorescent microscopy and flow cytometry were employed to detect the percentage of SP cells in THP-1 cells. Then, SP and non-SP (NSP) cell subpopulations were collected and identified. THP-1 cells were incubated with different concentrations of Ara-C for $24 \mathrm{~h}$ and the proportion of SP cells was detected. Our results demonstrated that the percentage of SP cells was $1.81 \pm 0.99 \%$ in THP-1 cells. A majority of the SP cells remained in the $G_{0} / G_{1}$ phase, and the expression of $\mathrm{CD}^{+}{ }^{+}$and $\mathrm{CD}_{34}{ }^{+} \mathrm{CD} 38^{-}$and the proliferation ability of the SP cells were higher compared to NSP cells $(\mathrm{P}<0.05)$. The mRNA expression of multidrug resistance genes (ABCG2 and $A B C B 1)$, apoptosis regulation genes $(B c l-2)$ and the $B c l-2 /$ $B a x$ value of SP cells were higher than those of NSP cells. SP cells have been shown to be more tumorigenic than NSP cells. Following co-culture with Ara-C, the proportion of SP cells increased significantly and subsequently the Ara-C concentration increased. These findings suggest that the THP-1 cell line contains SP cells and that SP cells possess certain intrinsic stem cell properties and may contain a larger proportion of leukemia stem cells (LSCs). The concentrations of SP cells can be increased with Ara-C by co-culture, and this technique is a useful and important application for the study of LSCs.
\end{abstract}

\section{Introduction}

Childhood and adolescent acute myeloid leukemia (AML) is one of the most challenging types of childhood cancer to successfully treat (1). The relapse rate remains unacceptably high, with a 5-year event-free survival (EFS) of approxi-

Correspondence to: Dr Yingchao Wang, Department of Pediatrics, The First Affiliated Hospital of Zhengzhou University, Zhengzhou, Henan 450052, P.R. China

E-mail: 314039104@qq.com

Key words: THP-1 cell line, leukemia stem cell, side population cell, arabinosylcytosine (Ara-C) mately $50 \%$ (2). In addition, successful treatment can only be achieved using highly intensive chemotherapy that results in relatively high rates of treatment-related mortality and significant side-effects (3). Although extensive efforts are being made to eliminate these issues, an efficient and effective method of treating AML has yet to be developed. Novel therapeutic strategies are urgently required to improve the prognosis of this disease.

The cancer stem cells (CSCs) theory postulates the origin of cancer from a new perspective. CSCs are a small subset of cancer cells that possess stem cell-like properties, such as, the ability to self-renew via asymmetric division and to produce differentiated progeny. These cells generally remain in a quiescent state (4) and comprise a small minority of the total tumor population. They have an extensive capacity to proliferate, differentiate, and self-renew, enabling them to repopulate recipients after transplantation (5). This small population of cells within a cancer is responsible for drug resistance and the recurrence of cancer (6). Hence, the specific targeting of CSCs therapeutically must be explored. To date, the possible existence of CSCs has been shown in leukemia $(7,8)$ and in certain solid tumors $(9,10)$. CSCs have also been identified in immortalized cell lines (5), long-term cultured cancer cells $(10,11)$, and patient tumor samples (5), using the side population (SP) technique.

Extensive research has focused on leukemia stem cells (LSCs) in the pursuit of new ideas for targeted therapy. Therefore, sorting, identifying and enriching LSCs has become particularly important in leukemia treatment research. Side population (SP) cells, as defined by Hoechst 33342 exclusion in flow cytometry, represent only a small fraction of the whole cell population (12); their properties occupy an important position in several investigations $(13,14)$. Previous studies have shown that CSCs can be identified by an SP phenotype based on fluorescence-activated flow cytometry. SP cells have been found not only in patient tumor samples but also in immortalized cell lines and long-term cultured cancer cells $(15,16)$; they have demonstrated the capacity to function as stem cells in the tissues from which they were isolated and may be able to transdifferentiate (17). SP cells share the most relevant features of LSCs, i.e., the self-renewal potential and quiescent status, and they contain relatively high concentrations of tumor stem cell indicators (18). Concurrent studies have shown that SP cells in human cancer have various origins, including acute myelogenous leukemia, neuroblastoma, and glioma $(10,11,19)$. These 
studies have also suggested that SP cells may be a source of cancer stem cells (CSCs). SP cells can be sorted using flow cytometry, which is a suitable application for LSC sorting (20).

The human acute monocytic leukemia cell line THP-1, which was originally established from an infant diagnosed with AML (21), provides an experimental model for functional, preclinical therapeutics and target identification studies of AML. In this study, we identified cancer SP cells by isolating them in the THP-1 cell line. In SP and NSP cells, we evaluated the cell cycle, the capacity for self-renewal, the presence of leukocyte surface antigens, and the expression of the multidrug resistance gene and the apoptosis gene. The aim of this study was to enrich the LSC subpopulation in the THP-1 cell line with arabinosylcytosine (Ara-C) and to study the relationship between SP cells and LSCs.

\section{Materials and methods}

Cell line and culture. The human acute monocytic leukemia cell line THP-1 (Shanghai Institute of Cell Biology) was cultured in RPMI-1640 medium (HyClone, Logan, UT, USA) supplemented with $10 \%$ fetal bovine serum (FBS) (Hyclone), $100 \mathrm{U} / \mathrm{ml}$ penicillin-streptomycin (Invitrogen Life Technologies, Grand Island, NY, USA) at $37^{\circ} \mathrm{C}$ under a $5 \%$ $\mathrm{CO}_{2}$ atmosphere.

SP cell analysis using fluorescence microscopy. The SP cells were suspended at $1 \times 10^{6}$ cells $/ \mathrm{ml}$ in pre-warmed RPMI-1640 medium containing $2 \% \mathrm{FBS}, 100 \mathrm{U} / \mathrm{ml}$ penicillin-streptomycin $\mathrm{G}, 100 \mu \mathrm{g} / \mathrm{ml}$ streptomycin and $10 \mathrm{mmol} / 1 \mathrm{HEPES}$ buffer. These cells were then incubated at $37^{\circ} \mathrm{C}$ for $90 \mathrm{~min}$ with $5 \mu \mathrm{g} / \mathrm{ml}$ Hoechst 33342 (Sigma-Aldrich, St. Louis, MO, USA), protected from light, either alone or in the presence of $50 \mu \mathrm{mol} / 1$ verapamil (Sigma-Aldrich). The cells were placed immediately on ice and were then washed and resuspended in cold phosphate-buffered saline (PBS) containing 1\% FBS. Fluorescence microscopy (Leica, Germany) was employed to detect the morphology of SP cells among the THP-1 cells. The cells that were not stained or colored light blue were identified as SP cells.

SP cell analysis and sorting using flow cytometry. Following incubation with Hoechst 33342 at $4^{\circ} \mathrm{C}, 1 \mu \mathrm{g} / \mathrm{ml}$ propidium iodide (PI) (BD Pharmingen, San Diego, CA, USA) was added to label the dead cells, and the mixture was then filtered through a $40-\mu \mathrm{m}$ cell strainer (BD Falcon) to obtain a singlecell suspension. Cell analyses and purification were performed using MoFlo carrying a triple-laser (DakoCytomation, Fort Collins, CO, USA). Hoechst 33342 was excited with the UV laser at $350 \mathrm{~nm}$, and fluorescence emission was measured with 405/BP30 (Hoechst blue) and 570/BP20 (Hoechst red) optical filters. PI labeling was measured through the 630/BP30 filter for the discrimination of dead cells. SP and NSP cells in each well were isolated (22).

Cell surface immunophenotyping. Immunophenotyping was conducted using conjugated monoclonal human antibodies reactive to $\mathrm{CD} 34$ and $\mathrm{CD} 38$ (BD Pharmingen). The staining was performed in the dark at $4^{\circ} \mathrm{C}$ for $30 \mathrm{~min}$. Isotype control antibodies and live unstained cells were used to establish
Table I. Primer sequences for different genes.

\begin{tabular}{ll}
\hline Gene & \multicolumn{1}{c}{ Primer sequences (5'-3') } \\
\hline GAPDH & F: ACCACAGTCCATGCCATCAC \\
& R: TCCACCACCCTGTTGCTGTA \\
ABCG2 & F: GTCTAAGCAGGGACGAACAATC \\
& R: GCCAATAAGGTGAGGCTATCAA \\
ABCB1 & F: TGGTGTTTGGAGAAATGACAGAT \\
& R: GAAACCTGAATGTAAGCAGCAAC \\
Bcl-2 & F: GTGGATGACTGAATACCTGAACC \\
& R: AGACAGCCAGGAGAAATCAAAC \\
Bax & F: GGTTGTCGCCCTTTTCTACTT \\
& R: GTGAGGAGGCTTGAGGAGTCT
\end{tabular}

F, forward; R, reverse.

gating parameters for positive cells. The percentage of the positive cells was obtained via the CellQuest software (BD Pharmingen).

Cell cycle analysis. SP and NSP cells were harvested and then washed twice with PBS. The supernatant was discarded and the pellets were dissolved with $1 \mathrm{ml}$ of $70 \%$ cold ethanol. After incubating at $4^{\circ} \mathrm{C}$ for at least $12 \mathrm{~h}$, cells were stained with $50 \mathrm{~g} / \mathrm{ml}$ PI supplemented with $50 \mathrm{~g} / \mathrm{ml}$ RNase and then incubated in the dark at $21^{\circ} \mathrm{C}$ for $30 \mathrm{~min}$. The samples were profiled for DNA content by flow cytometry (BD Biosciences), and 10,000 events were recorded for each sample. The percentages of cells in the $G_{0} / G_{1}, S$ and $G_{2} / M$ phases were obtained by CellQuest software.

Cell proliferation assay. The cells were grown in a 96-well plate, and the relative cell number was determined using a Cell Counting Kit-8 (Dojindo, Kumamoto, Japan) according to the manufacturer's protocol. The SP and NSP cells were plated at a density of $1 \times 10^{4}$ cells/well in 96-well plates for 0-9 days. After $10 \mu \mathrm{l}$ CCK-8 solution was added to each well, cells were incubated for a further $4 \mathrm{~h}$ at $37^{\circ} \mathrm{C}$, and the absorbance was measured at $450 \mathrm{~nm}$ using an automated ELISA reader (BioTek, Winooski, VT, USA).

Validation of gene expression by $q P C R$. Quantitative-PCR (qPCR) analysis was performed according to the manufacturer's protocol. First, total RNA was extracted from cells with an RNAprep pure Cell kit (Tiangen Biotech Co., Ltd., Beijing, China). One microgram of total RNA was reverse transcribed into cDNA with $1 \mu \mathrm{l}$ M-MuLV RT (200 $\mu \mathrm{g} / \mu \mathrm{l})$ using a Single-Strand cDNA Synthesis Kit (Stratagene, La Jolla, CA, USA) and analyzed using an ABI 7900 (Applied Biosystems, Foster City, CA, USA). Specific primers for qPCR of GAPDH (housekeeping gene) and the additional genes of interest were designed using Assay-by-Design primer design software (Applied Biosystems) or were purchased as Assayson-Demand from Applied Biosystems. The primers for these genes are shown in Table I. The relative amounts of product were calculated using the comparative CT $\left(2^{-\Delta \Delta \mathrm{Ct}}\right)$ method. 


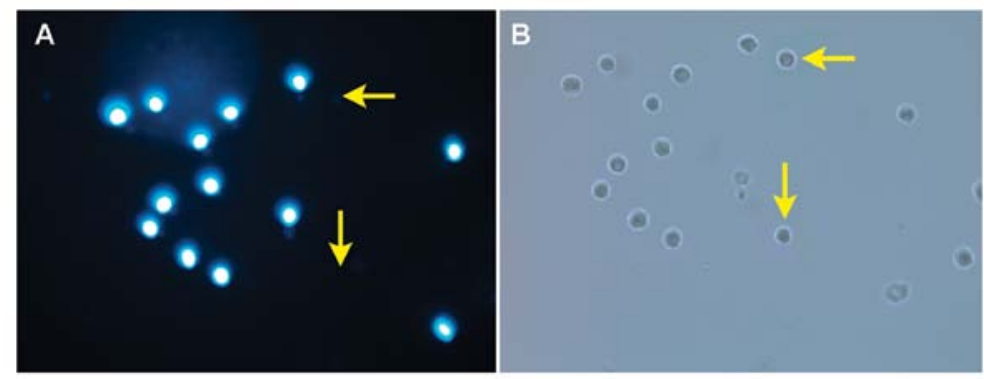

Figure 1. Hoechst 33342 staining of THP-1 cells (magnification, x200). Micrographs of THP-1 cells under a fluorescence microscope. (B) Micrographs of THP-1 cells under a common microscope (magnification, x200). The cells indicated by the arrow are SP cells.
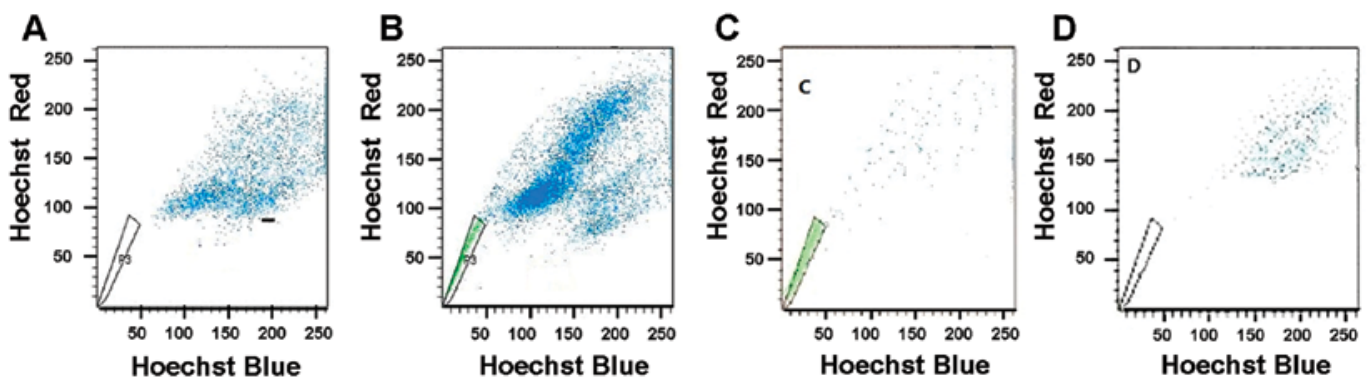

Figure 2. Cell sorting results and sorting purity. (A) The SP cells appear as the Hoechst-resistant fraction capable of pumping out the dye, and typically represent $1.81 \pm 0.99 \%$ of viable cells from the THP-1 leukemia cell line. The NSP cells that retained high levels of Hoechst staining are the main population of cells. (B) The SP is ablated when verapamil is included in the Hoechst incubation. (C) The sorted SP cells were concentrated in the SP region, the purity reached $96.75 \pm 1.55 \%$. (D) The NSP tube cells were concentrated in the subregion of the main group of cells prior to the election, the purity reached $97.03 \pm 1.87 \%$.

In vivo tumor formation. All animal studies were performed in compliance with the Guidelines for the Care and Use of the Laboratory Animals in Henan Province, China. Naïve male 6-8-week-old NOD/SCID mice were obtained from Beijing HFK Bioscience Co. (Beijing, China), kept under specific pathogen-free (SPF) conditions and used as tumor transplant recipients. Mice were housed five per cage. Thirty mice were randomly divided into six groups, five mice per group. Growing cells, sorted from the SP cells $\left(1 \times 10^{3}, 1 \times 10^{4}\right.$ and $1 \times 10^{5}$ per mouse) and NSP cells $\left(1 \times 10^{4}, 1 \times 10^{5}\right.$ and $1 \times 10^{6}$ per mouse) diluted in PBS, were mixed with $50 \mathrm{ml}$ Matrigel (BD Biosciences) and injected intravenously via the tail vein. Three to four weeks later, human AML engraftment (hCD45 ${ }^{+}$ $\mathrm{CD}_{3}{ }^{+}$cells) was assessed in the peripheral blood and bone marrow by tail bleed and aspiration of the femur, respectively.

Enrichment of LSCs in an SP of THP-1 with Ara-C. THP-1 cells $\left(1 \times 10^{8}\right.$ cells $\left./ \mathrm{ml}\right)$ were incubated for $24 \mathrm{~h}$ at $37^{\circ} \mathrm{C}$ under a $5 \% \mathrm{CO}_{2}$ atmosphere with four different concentrations of Ara-C: $10,100,1,000$ and 2,000 $\mu \mathrm{g} / \mathrm{ml}$. The cells were then harvested and washed twice with PBS. The proportion of SP cells was detected, respectively, for each Ara-C concentration.

Statistical analysis. Statistical analyses were performed using SPSS 17.0 (SPSS, Inc., Chicago, IL, USA). Data are presented as the means $\pm \mathrm{SD}$. Differences were determined using the Student's t-test or Fisher's exact test and one-way analysis of variance (ANOVA) followed by Scheffe's post hoc test, as indicated in the text. $\mathrm{P}<0.05$ was considered to indicate a statistically significant difference. All experiments were performed in triplicate.

\section{Results}

Prevalence of SP cells in THP-1 cells. The SP cells overexpressed the multidrug-resistant proteins that allowed them to efflux various drugs and xenobiotics, as well as the Hoechst 33342 dye (23). As shown in Fig. 1, fluorescence microscopy with Hoechst 33342 staining demonstrated that the SP cells were present among the THP-1 cells. Whereas $>90 \%$ of the THP-1 cells were stained intense blue, a small population of the cells remained unstained (Fig. 1A, arrow). The micrographs of THP-1 SP and NSP cells were observed under visible light. There was no significant difference between SP and NSP cells in morphology (Fig. 1B, arrow).

Flow cytometry analysis with Hoechst 33342 staining demonstrated that the dimly stained Hoechst 33342 cells on the corner of the plot were gated as the SP population and represented a percentage of $1.81 \pm 0.99 \%$ of total THP-1 cells (Fig. 2A). Since the SP profile was blocked by staining in the presence of verapamil, a calcium channel blocker, the SP cell frequency was practically eliminated in the THP-1 cells stained with Hoechst 33342 and verapamil (Fig. 2B). To determine the sensitivity of our system, the SP and NSP cells in THP-1 cells were sorted and the purity was tested separately. The results showed that the cells of SP tube were still concentrated in the THP-1 SP region and the cells of NSP tube were still concentrated in the subregion of the main group of cells prior to sorting. Purity levels were $96.75 \pm 1.55 \%$ and $97.03 \pm 1.87 \%$, respectively (Fig. 2C and D).

Expression of cell surface markers in SP and NSP cells. To further define the cells within the SP fraction, we used two 

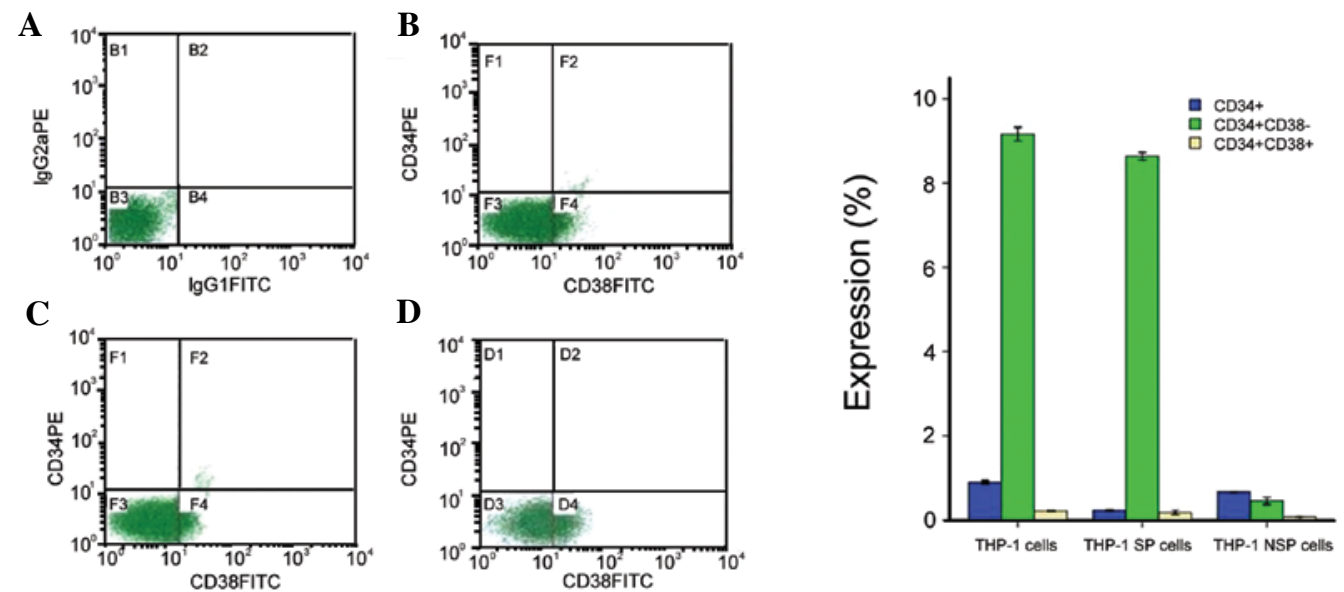

Figure 3. Flow cytometry analysis of CD34/CD38 expression. (A) IgG isotype control in THP-1 cells. (B) CD34/CD38 expression in unsorted THP-1 cells. (C) CD34/CD38 expression in the SP fraction. (D) CD34/CD38 expression in the NSP fraction.
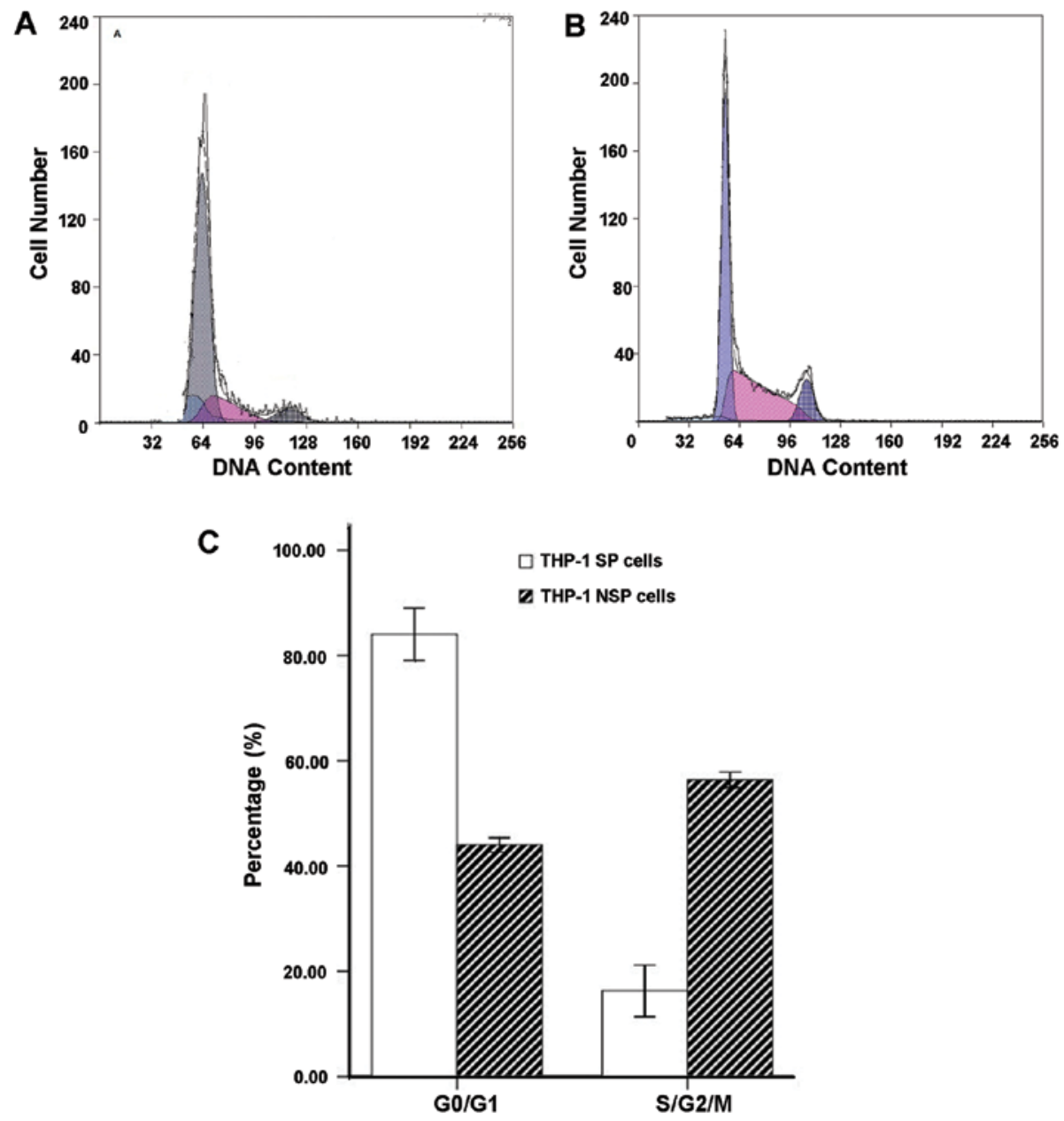

Figure 4. Cell cycle analysis by flow cytometry. (A) Cell cycle distribution in the SP cell groups. (B) Cell cycle distribution in the NSP cell groups. (C) Results of duplicate determinations from three separate experiments. ${ }^{*} \mathrm{P}<0.05$ vs. SP cells.

cell-surface markers (CD34 and CD38) associated with LSCs (24). The analysis of SP and NSP cells revealed that these cells differ significantly in the expression of cell surface markers. The overall percentage of cells positive for CD34 was significantly lower in the NSP compared with the SP cells in all cell lines examined (Fig. 3).
Although the expression of $\mathrm{CD} 34^{+} / \mathrm{CD} 38^{-}$in the SP fraction (Fig. 3C) was small, it was statistically significant and substantially higher than that in unsorted THP-1 cells (Fig. 3B) and NSP cells (Fig. 3D). The percentage of CD $34^{+}$and $\mathrm{CD} 38^{-}$ expression in SP cells was $8.68 \pm 0.20 \%$, markedly higher than that in NSP cells $(0.16 \pm 0.08 \%)(\mathrm{P}<0.05)$ (Fig. 3). 


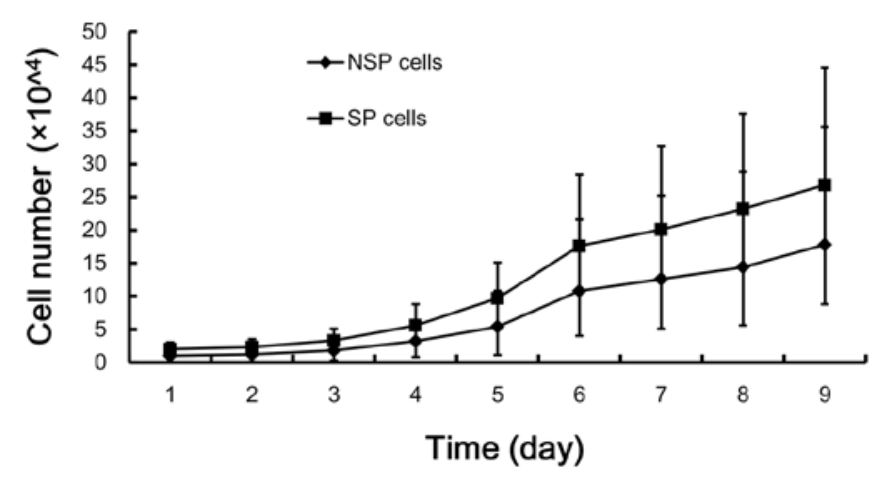

Figure 5. Growth curve of THP-1 SP and NSP cells. The THP-1 SP cells under stem cell-selective conditions grew faster compared with THP-1 NSP cells $(\mathrm{P}<0.05)$.

Cell cycle in THP-1 SP and NSP cells. The $\mathrm{G}_{0} / \mathrm{G}_{1}$ phase cells in the THP-1 SP subpopulation accounted for $\sim 84.04 \pm 4.98 \%$ of the total cells and more than NSP cells $(44.02 \pm 1.35 \%)$.

THP-1 SP cells resulted in blockage of the cell cycle from the $\mathrm{G}_{0} / \mathrm{G}_{1}$ phase to the $\mathrm{S}$ phase. Compared with NSP cells, the ratio of $\mathrm{G}_{0} / \mathrm{G}_{1}$ phase cells significantly increased, and the ratio of S-phase cells significantly decreased in SP cells $(\mathrm{P}<0.05)$ (Fig. 4).

In vivo growth characteristics of SP and NSP cells. The growth characteristics of the SP subpopulation were consistent with the predicted behavior of primitive precursor cells, including a high proliferative rate and self-renewal capacity. The proliferation of SP cells was significantly higher than that of NSP cells $(\mathrm{P}<0.05)$ (Fig. 5).

Analysis of gene expression in SP and NSP cells. We isolated RNA from sorted SP and NSP cells of the leukemia cell line THP-1 and used quantitative real-time RT-PCR amplification analysis to quantify the relative expression of the $\mathrm{ABC}$ transporter gene, the $A B C B 1$ and $A B C G 2$ gene product currently believed to be most closely associated with the SP phenotype (25). All SP fractions expressed higher levels of the $A B C B 1$ and $A B C G 2$ transporter gene than did the NSP fractions (Fig. 6A). We also measured the expression levels of two other cell apoptosis genes, Bcl-2 and Bax; the former gene was clearly expressed at higher concentrations in SP compared with the NSP, whereas the latter gene was not. However, the $B c l-2$ and Bax values were significantly higher than in the NSP $(\mathrm{P}<0.05)$ (Fig. 6B).

THP-1 SP cells exhibit higher tumorigenicity than NSP cells. To determine whether the SP cells we identified in the THP-1 cell line might also be more tumorigenic, we performed xenograft experiments in vivo. Three or 4 weeks after THP-1 SP cells were transplanted intravenously via the tail vein, human AML engraftment $\left(\mathrm{hCD} 45^{+} / \mathrm{CD} 33^{+}\right)$was assessed in the peripheral blood and bone marrow by tail bleed and aspiration of the femur, respectively. The results indicated that the systemic disseminated leukemia model had been established successfully by injecting $1 \times 10^{3}$ THP-1 SP cells in NOD/SCID mice (Fig. 6A). The SP cells isolated from the THP-1 cells were more tumorigenic than the NSP cells. NSP cells require a quantity of at least $1 \times 10^{6}$ to establish a tumor model. Statistically, there were significant differences in the incidence of leukemia among different groups $(\mathrm{P}<0.05)$. The H\&E strain of histologic sections revealed that almost all the leukemia xenotransplants successfully induced leukemia in the mice (Fig. 7B and C).

SP increases in the THP-1 cell line. It has been reported that CSCs can resist apoptosis when they are exposed to apoptosis inducement factors (26). Considering this characteristic of CSCs, we formulated a hypothesis that apoptosis resistance of CSCs can be used to increase the proportion of SP cells. In an apoptosis inducement model, the proportion of SP cells may increase in surviving cells. In this study, SP cells were co-cultured with different concentrations of Ara-C, the proportion of SP cells increased significantly, and the proportion of SP cells increased with the Ara-C concentration (Fig. 8).
A

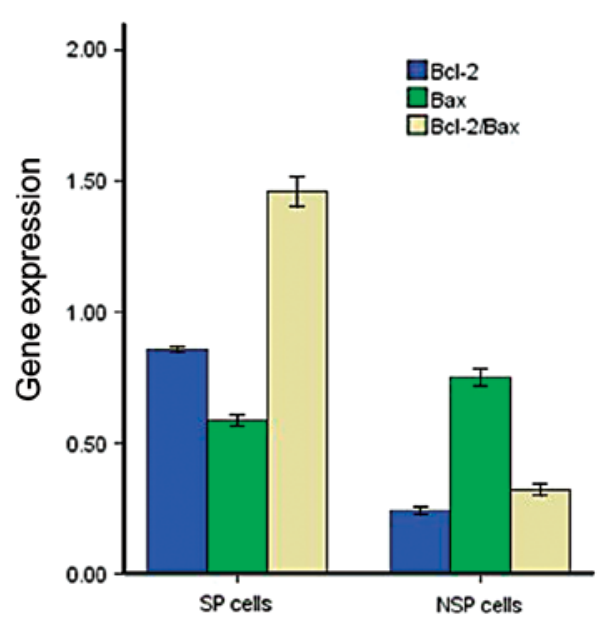

B

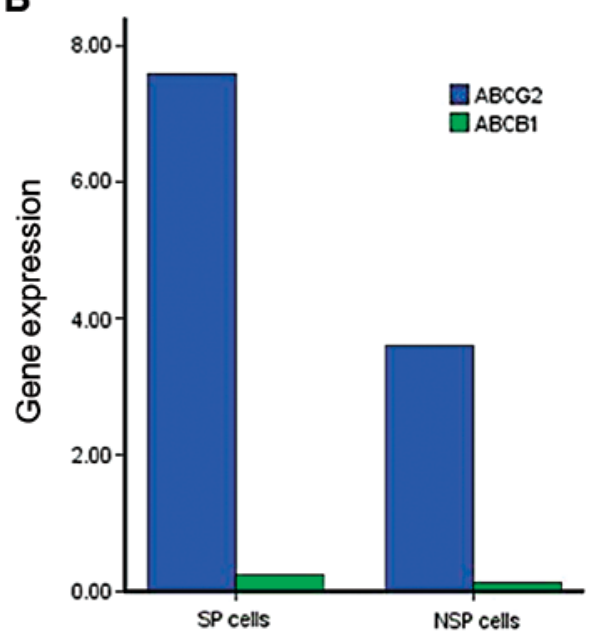

Figure 6. Analysis of ABCB1, ABCG2, Bcl-2 and Bax gene expression. 
A
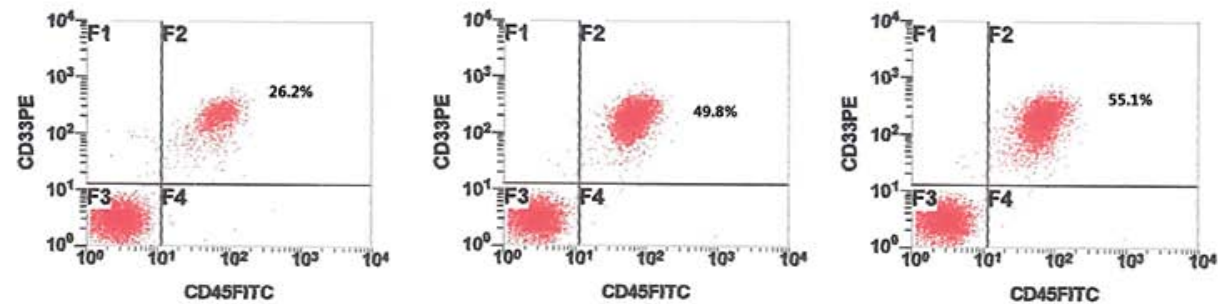

B
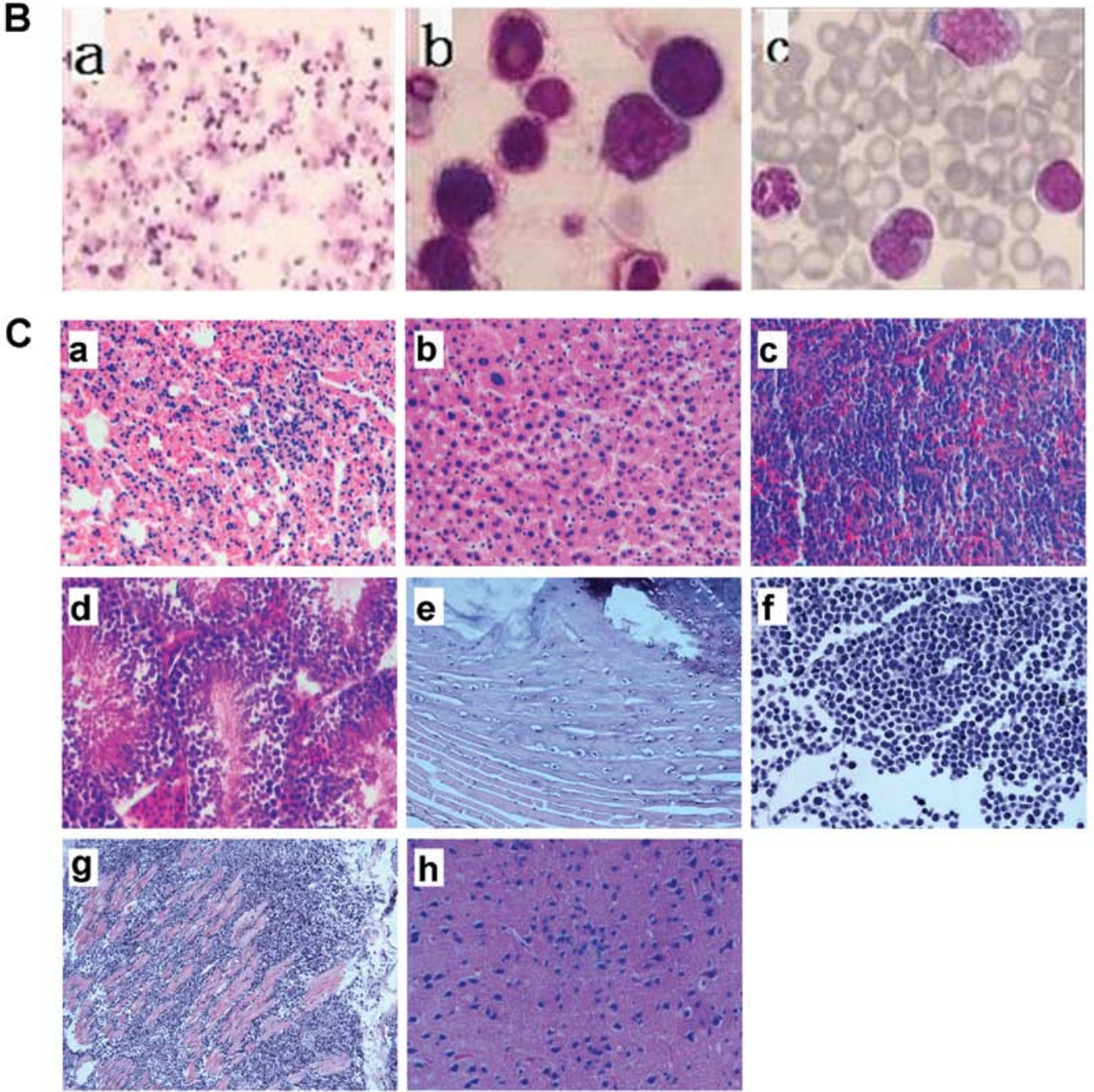

Figure 7. Identification of human THP-1 xenotransplant leukemia models. (A) Human AML engraftment (hCD45+CD33+ cells) was assessed in the peripheral blood and bone marrow using flow cytometry. (B) Wright's staining of bone marrow of NOD/SCID mice injected with SP cells. (a) magnification, x100, (b) magnification, $\mathrm{x} 1,000$ and (c) peripheral blood (magnification, $\mathrm{x} 1,000)$. (C) Pathological examination of NOD/SCID mice injected with SP cells. Representative H\&E staining sections of organs and tissues (H\&E magnification, x200). AML cells can be found in (a) lung, (b) liver, (c) spleen, (d) didymus, (e) paravertebral muscles, (f) lymph node, and (g) musculi faciales. (h) Cerebral edema was observed.
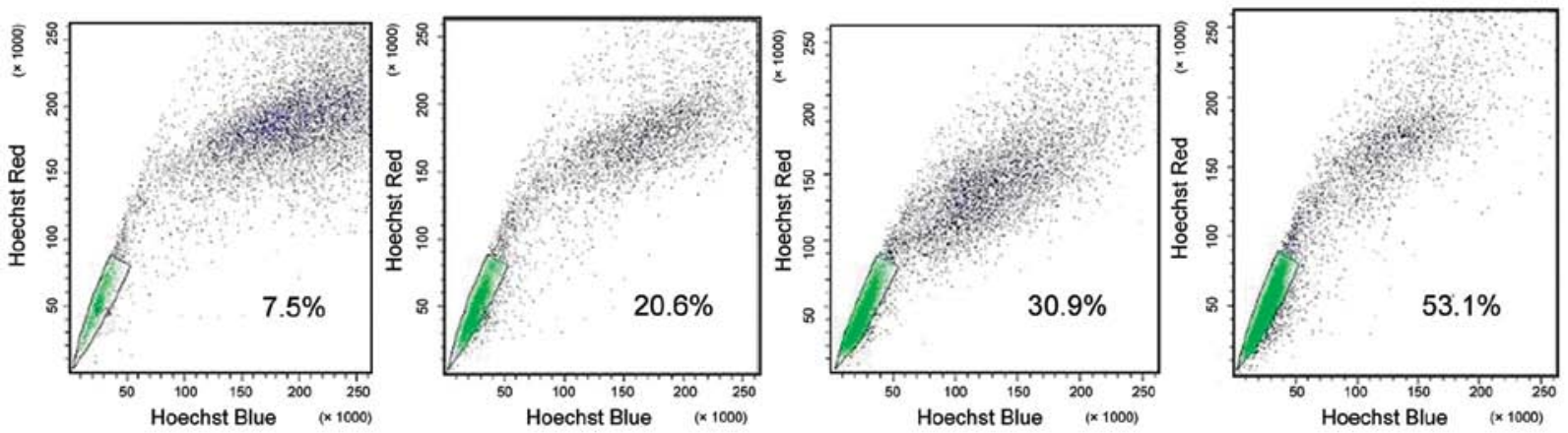

Figure 8. The proportion of SP cells after treatment with different concentrations of Ara-C. Following co-culture with different concentrations of Ara-C, the proportion of SP cells increased significantly and the proportion of SP cells increased with Ara-C concentration. 


\section{Discussion}

Using the side population (SP) technique, Zheng et al (27) reported the SP rate in acute promyelocytic leukemia NB4 cells to be less than 1\%. In addition, SP cells possess intrinsic stem cell properties and express some of the characteristic stem cell genes. In the present study, analysis by fluorescence microscopy and flow cytometry demonstrated the presence of SP cells, and we were able to identify a small SP component $(1.81 \pm 0.99 \%)$ of cancer cells from the THP-1 leukemia cell line. The SP cells were practically non-existent in the presence of Hoechst 33342 and verapamil, a calcium channel blocker. The percentages of SP cells detected were similar to those in most previous reports: $0.8-1.9 \%$ in human multiple myeloma cell line RPMI-8226 and NCI-H929 (28), $0.47-4 \%$ in an adult T-cell leukemia/lymphoma cell line (29), and $0.5-29.9 \%$ in human acute myeloid leukemia (AML) (8), but less than the 4-37\% noted in neuroblastoma cell lines (15). Furthermore, the SP cells and the majority of non-SP (NSP) cells were indistinguishable morphologically. Therefore, the isolation and identification of cancer stem cells (CSCs) remains very difficult (8). To the best of our knowledge, this is the first described isolation of cancer stem-like cells from the THP-1 cell line.

To date, AML leukemia stem cells (LSCs) are the most well-studied CSCs population (30). AML is typically a disease of stem progenitor cell origin. No special markers have been successfully developed to identify those cells in different tumors; different tumors have different CSCs markers (one or more). Blair et al (31) demonstrated that only a small quantity of a defined subset of cells were consistently clonogenic and that all AML LSC subtypes possess the same cell-surface markers (32). As determined by immunophenotyping, SP cells share some phenotypic characteristics with bone marrow, such as the absence of mature hematopoietic lineage markers CD34 and expression of CD38. Bonnet et al have identified LSCs in human AML as a common immunophenotype (CD34 $\left.{ }^{+} / \mathrm{CD} 38^{-}\right)$ and have demonstrated their self-renewal potential (1). We therefore examined the expression of CD34/CD38 in SP cells, NSP cells, and unsorted THP-1 cells. Our results showed that CD34/CD38 were both expressed at low levels on the membranes of SP and NSP cells. The percentages of CD34 ${ }^{+}$ and $\mathrm{CD} 34^{+} / \mathrm{CD} 38^{-}$cells in the SP were higher than those in the NSP and among common THP-1 cells $(\mathrm{P}<0.05)$. These results suggest that the presence of LSCs in THP-1 cells is rare and that the number of LSCs is quite limited; however, LSCs may be more prevalent in the SP.

LSCs, similar to their normal HSC counterparts, exhibit a range of characteristics that enable their long-term survival. Some of these characteristics also facilitate their escape from the cytotoxic effects of chemotherapy; for example, LSCs are primarily present in a quiescent phase of the cell cycle (33). In the present study, the cell cycles of two cell subsets, SP and NSP of the THP-1 cell line, were analyzed by flow cytometry. A majority of the SP cells remained in the $\mathrm{G}_{0} / \mathrm{G}_{1}$ phase, and the NSP cells remained in the $S$ or $G_{2}$ or $M$ phases. The proliferation of SP cells was significantly higher than that of NSP cells $(\mathrm{P}<0.05)$.

A study by Hope et al (34) showed that AML originates from a hierarchy of LSC classes that differ in self-renewal capacity. Clarke et al (35) reported that SP cells produced two to seven times more colonies than NSP cells. In support of their putative stem cell nature, only the SP cells possessed the ability to produce colonies with both myoepithelial and luminal epithelial cell types. In the present study, the cell growth curves showed that the growth rate of SP cells was significantly faster than that of NSP cells; the cells continued to proliferate and the plateau period was not evident. This observation may indicate that SP cells contain more LSCs, and, therefore, cell proliferation is accelerated.

SPs are small subpopulations of cells with enriched stem cell activity that show a distinct 'low' Hoechst 33342 dye-staining pattern. The SP phenotype is mediated by the ATP-binding cassette $(\mathrm{ABC})$ family of transporter proteins. Various types of $A B C$ transporters have been shown to contribute to drug resistance in numerous types of cancer by pumping drugs out of cells $(25,36)$. Notably, some ABC transporters are expressed by several types of stem cells. One of the major mediators seems to be ABCG2 or BCRP (37), which was initially identified in drug-selected MCF7 breast cancer cells and was later found to efflux multiple chemotherapeutic drugs and xenobiotics (38). Other supporting evidence shows that SP cells preferentially express ABCG2 $(13,39)$. SP cells also express other $\mathrm{ABC}$ transporters such as MDR-1 (i.e., ABCB1 or P-glycoprotein), suggesting that these latter molecules may also be involved in mediating the SP phenotype (40). We therefore isolated RNA from sorted SP and NSP cells of the THP-1 leukemia cell lines, and we used a real-time RT-PCR assay to quantify the relative expression of the ABCG2 and ABCB1 transporters. The mRNA expression levels of the two genes of SP cells were higher than those among NSP cells. Independent of whether THP-1SP cells are indeed a tumor 'stem cell' population, their high expression of drug efflux transporter genes and their associated high capacity to efflux lipophilic drugs may have a significant effect on treatment outcome (5).

Stem cell resistance to apoptosis through complicated mechanisms $(41,42)$, such as the regulation of Bcl-2 or Bax $(43,44)$, has been proven experimentally. Compared with SP cells, NSP cells in tumors are more susceptible to apoptosis inducement or chemotherapy. We also measured the expression levels of two other apoptosis regulation genes, Bcl-2 and Bax; the former gene was clearly expressed at higher concentrations in SP cells compared with NSP cells, whereas the latter gene was not. There was no difference between SP and NSP cells in Bax expression, but the Bcl-2/Bax values of the SP cells were significantly higher than those of the NSP cells. These results suggest that apoptosis resistance may aid in screening the markers of CSCs.

It is believed that only the CSCs, but not the majority of their remaining descendants, are responsible for tumorigenesis, progression, metastasis, and relapse following treatment (45). Repopulation in recipients after transplantation is the most important characteristic of CSCs. Purified SP cells from certain cell lines were more tumorigenic than the corresponding NSP cells. To evaluate the tumorigenic ability of THP-1 SP cells in vivo, NOD/SCID mice were injected with different subpopulations and different quantities of THP-1 cells intravenously by tail vein, and the incidence of leukemia was compared among the groups. As we anticipated, the systemic disseminated leukemia model was established successfully by 
injecting 1x10 3 THP-1 SP cells. Unsorted THP-1 cells require a quantity of at least $1 \times 10^{6}$ to establish a tumor model.

Isolation and identification of the SP cells is helpful in studying the difference between the CSCs and nontumorigenic cells in certain aspects. However, the proportion of LSCs is quite limited, and the isolation and identification of CSCs is very difficult. CSCs have been reported to resist apoptosis when they are exposed to apoptosis inducement factors (26). This characteristic of CSCs led us to formulate a hypothesis that apoptosis resistance by CSCs can be used to increase the proportion of SP cells. In an apoptosis inducement model, the proportion of SP cells may increase in surviving cells. Cytarabine (Ara-C) is commonly used for the treatment of acute leukemia. Incorporation of Ara-C into DNA is a key event in the killing of proliferating leukemic cells, but it is relatively ineffective against LSCs, which retain a quiescent status (46). In this study, we used Ara-C to kill common proliferating THP-1 cells. Following co-culture with Ara-C, the proportion of SP cells increased significantly and with Ara-C concentration.

In our study, all the representative LSC markers were significantly increased in SP cells compared with NSP cells; therefore, our results suggest that isolated SP cells could characterize the properties of LSCs. The proportion of SP cells may be increased after they are co-cultured with Ara-C, and this technique can be applied to the study of LSCs.

\section{References}

1. Woods WG: Curing childhood acute myeloid leukemia (AML) at the half-way point: promises to keep and miles to go before we sleep. Pediatr Blood Cancer 46: 565-569, 2006.

2. Gorman MF, Ji L, Ko RH, et al: Outcome for children treated for relapsed or refractory acute myelogenous leukemia (rAML): a Therapeutic Advances in Childhood Leukemia (TACL) Consortium study. Pediatr Blood Cancer 55: 421-429, 2010.

3. Kaspers GJ and Zwaan CM: Pediatric acute myeloid leukemia: towards high-quality cure of all patients. Haematologica 92 1519-1532, 2007.

4. Reya T, Morrison SJ, Clarke MF and Weissman IL: Stem cells, cancer, and cancer stem cells. Nature 414: 105-111, 2001.

5. Benchaouir R, Rameau P, Decraene C, et al: Evidence for a resident subset of cells with SP phenotype in the $\mathrm{C} 2 \mathrm{C} 12$ myogenic line: a tool to explore muscle stem cell biology. Exp Cell Res 294 254-268, 2004

6. Schatton T, Murphy GF, Frank NY, et al: Identification of cells initiating human melanomas. Nature 451: 345-349, 2008.

7. Lapidot T, Sirard C, Vormoor J, et al: A cell initiating human acute myeloid leukaemia after transplantation into SCID mice. Nature 367: 645-648, 1994.

8. Bonnet D and Dick JE: Human acute myeloid leukemia is organized as a hierarchy that originates from a primitive hematopoietic cell. Nat Med 3: 730-737, 1997.

9. Al-Hajj M, Wicha MS, Benito-Hernandez A, Morrison SJ and Clarke MF: Prospective identification of tumorigenic breast cancer cells. Proc Natl Acad Sci USA 100: 3983-3988, 2003.

10. Kondo T, Setoguchi T and Taga T: Persistence of a small subpopulation of cancer stem-like cells in the C6 glioma cell line. Proc Natl Acad Sci USA 101: 781-786, 2004.

11. Hirschmann-Jax C, Foster AE, Wulf GG, et al: A distinct 'side population' of cells with high drug efflux capacity in human tumor cells. Proc Natl Acad Sci USA 101: 14228-14233, 2004.

12. Goodell MA, Brose K, Paradis G, Conner AS and Mulligan RC: Isolation and functional properties of murine hematopoietic stem cells that are replicating in vivo. J Exp Med 183: 1797-1806, 1996.

13. Shimano K, Satake M, Okaya A, et al: Hepatic oval cells have the side population phenotype defined by expression of ATP-binding cassette transporter ABCG2/BCRP1. Am J Pathol 163: 3-9, 2003
14. Falciatori I, Borsellino G, Haliassos N, et al: Identification and enrichment of spermatogonial stem cells displaying side-population phenotype in immature mouse testis. FASEB J 18: 376-378, 2004.

15. Hu C, Li H, Li J, et al: Analysis of ABCG2 expression and side population identifies intrinsic drug efflux in the $\mathrm{HCC}$ cell line MHCC-97L and its modulation by Akt signaling. Carcinogenesis 29: 2289-2297, 2008.

16. Wang J, Guo LP, Chen LZ, Zeng YX and Lu SH: Identification of cancer stem cell-like side population cells in human nasopharyngeal carcinoma cell line. Cancer Res 67: 3716-3724, 2007.

17. Haraguchi $\mathrm{N}$, Utsunomiya $\mathrm{T}$, Inoue $\mathrm{H}$, et al: Characterization of a side population of cancer cells from human gastrointestinal system. Stem Cells 24: 506-513, 2006.

18. Setoguchi T, Taga T and Kondo T: Cancer stem cells persist in many cancer cell lines. Cell Cycle 3: 414-415, 2004.

19. Feuring-Buske M and Hogge DE: Hoechst 33342 efflux identifies a subpopulation of cytogenetically normal CD34(+)CD38(-) progenitor cells from patients with acute myeloid leukemia. Blood 97: 3882-3889, 2001.

20. Guo Y, Follo M, Geiger K, Lubbert $M$ and Engelhardt $M$ : Side-population cells from different precursor compartments. J Hematother Stem Cell Res 12: 71-82, 2003.

21. Tsuchiya S, Yamabe M, Yamaguchi Y, Kobayashi Y, Konno T and Tada K: Establishment and characterization of a human acute monocytic leukemia cell line (THP-1). Int J Cancer 26: 171-176, 1980.

22. Gao Q, Geng L, Kvalheim G, Gaudernack G and Suo Z: Identification of cancer stem-like side population cells in ovarian cancer cell line OVCAR-3. Ultrastruct Pathol 33: 175-181, 2009.

23. Ishikawa $T$ and Nakagawa $H$ : Human $A B C$ transporter $A B C G 2$ in cancer chemotherapy and pharmacogenomics. J Exp Ther Oncol 8: 5-24, 2009.

24. Rombouts WJ, Martens AC and Ploemacher RE: Identification of variables determining the engraftment potential of human acute myeloid leukemia in the immunodeficient NOD/SCID human chimera model. Leukemia 14: 889-897, 2000.

25. Jonker JW, Freeman J, Bolscher E, et al: Contribution of the $\mathrm{ABC}$ transporters Bcrp1 and Mdrla/1b to the side population phenotype in mammary gland and bone marrow of mice. Stem Cells 23: 1059-1065, 2005.

26. Raguz S and Yague E: Resistance to chemotherapy: new treatments and novel insights into an old problem. Br J Cancer 99: 387-391, 2008.

27. Zheng X, Seshire A, Ruster B, et al: Arsenic but not all-trans retinoic acid overcomes the aberrant stem cell capacity of PML/RARalpha-positive leukemic stem cells. Haematologica 92: 323-331, 2007.

28. Matsui W, Wang Q, Barber JP, et al: Clonogenic multiple myeloma progenitors, stem cell properties, and drug resistance. Cancer Res 68: 190-197, 2008.

29. Kayo H, Yamazaki H, Nishida H, Dang NH and Morimoto C: Stem cell properties and the side population cells as a target for interferon- $\alpha$ in adult T-cell leukemia/lymphoma. Biochem Biophys Res Commun 364: 808-814, 2007.

30. Wang JC and Dick JE: Cancer stem cells: lessons from leukemia. Trends Cell Biol 15: 494-501, 2005.

31. Blair A, Hogge DE, Ailles LE, Lansdorp PM and Sutherland HJ: Lack of expression of Thy-1 (CD90) on acute myeloid leukemia cells with long-term proliferative ability in vitro and in vivo. Blood 89: 3104-3112, 1997.

32. Blair A and Sutherland HJ: Primitive acute myeloid leukemia cells with long-term proliferative ability in vitro and in vivo lack surface expression of c-kit (CD117). Exp Hematol 28: 660-671, 2000 .

33. Naka K, Hoshii T and Hirao A: Novel therapeutic approach to eradicate tyrosine kinase inhibitor resistant chronic myeloid leukemia stem cells. Cancer Sci 101: 1577-1581, 2010.

34. Hope KJ, Jin L and Dick JE: Acute myeloid leukemia originates from a hierarchy of leukemic stem cell classes that differ in selfrenewal capacity. Nat Immunol 5: 738-743, 2004.

35. Clarke RB: Isolation and characterization of human mammary stem cells. Cell Prolif 38: 375-386, 2005.

36. Chang XB: Molecular mechanism of ATP-dependent solute transport by multidrug resistance-associated protein 1 . Methods Mol Biol 596: 223-249, 2010.

37. Zhou S, Schuetz JD, Bunting KD, et al: The ABC transporter Bcrp1/ABCG2 is expressed in a wide variety of stem cells and is a molecular determinant of the side-population phenotype. Nat Med 7: 1028-1034, 2001. 
38. Doyle LA and Ross DD: Multidrug resistance mediated by the breast cancer resistance protein BCRP (ABCG2). Oncogene 22: 7340-7358, 2003.

39. Summer R, Kotton DN, Sun X, Ma B, Fitzsimmons K and Fine A Side population cells and Bcrpl expression in lung. Am J Physiol Lung Cell Mol Physiol 285: L97-L104, 2003.

40. Bunting KD, Zhou S, Lu T and Sorrentino BP: Enforced P-glycoprotein pump function in murine bone marrow cells results in expansion of side population stem cells in vitro and repopulating cells in vivo. Blood 96: 902-909, 2000.

41. Taipale $J$ and Beachy PA: The Hedgehog and Wnt signalling pathways in cancer. Nature 411: 349-354, 2001.

42. van Stijn A, van der Pol MA, Kok A, et al: Differences between the $\mathrm{CD}^{3} 4^{+}$and $\mathrm{CD} 34^{-}$blast compartments in apoptosis resistance in acute myeloid leukemia. Haematologica 88: 497-508, 2003.
43. Chipuk JE, Moldoveanu T, Llambi F, Parsons MJ and Green DR: The BCL-2 family reunion. Mol Cell 37: 299-310, 2010.

44. Zhang QL, Niu Q, Niu PY, et al: Bax gene silencing: a potential intervention in aluminum-induced neural cell death. J Biol Regul Homeost Agents 24: 7-17, 2010.

45. Komuro H, Saihara R, Shinya M, et al: Identification of side population cells (stem-like cell population) in pediatric solid tumor cell lines. J Pediatr Surg 42: 2040-2045, 2007.

46. Misaghian N, Ligresti G, Steelman LS, et al: Targeting the leukemic stem cell: the Holy Grail of leukemia therapy. Leukemia 23: 25-42, 2009. 